\title{
Preventing and Treating PTSD and Related Conditions in Adults: A Research Agenda
}

\author{
Catherine A. Forneris, Gerald Gartlehner, Daniel E. Jonas, and Kathleen N. Lohr
}

Post-traumatic stress disorder (PTSD) may develop

following exposure to an extreme traumatic stressor: directly experiencing an event that involves actual or threatened death, serious injury, or other threat to one's physical integrity; witnessing an event that involves death, injury, or a threat to the physical integrity of another person; or learning about unexpected or violent death, serious harm, or threat of death or injury experienced by a family member or other close

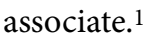

Lifetime prevalence of PTSD in the United States has been estimated at almost $7 \%$ (9.7\% in women; $3.6 \%$ in men); rates are higher for military personnel.2,3 In most people, posttraumatic symptoms resolve spontaneously in the first several weeks after the trauma. However, trauma exposure leads to PTSD in a substantial number of adults (10\% to $20 \%$ of those exposed).This condition can in turn lower educational attainment, impair role functioning (e.g., for work), reduce earnings, and produce familial and marital discord. Adult PTSD is associated with an increased likelihood of having another psychiatric disorder, most notably substance use disorders or major depressive disorder, and with an increased risk of suicide.

Preventing PTSD can potentially reduce a significant burden of individual and societal suffering. Preventive services involve psychological, pharmacological, and newer approaches such as complementary and alternative medicine. They are used individually and in combination, but little evidence supports their effectiveness.

Choosing PTSD treatments can also be uncertain. Recommendations are not typically based on evidence from systematic reviews of the literature; indeed, sometimes the recommendations are inconsistent. For adults, several psychological and pharmacological interventions are apparently effective. However, evidence comparing different therapies is scant and whether any approaches are more effective for victims of particular types of trauma is unknown.

\section{Key Findings}

- Post-traumatic stress disorder (PTSD) may develop following exposure to an extreme traumatic stressor in civilian or military life.

- Lifetime prevalence of PTSD among trauma-exposed adults in the United States has been estimated at almost 7\% (9.7\% in women; 3.6\% in men, with higher rates for military personnel).

- Persons exposed to traumatic events leading to either PTSD symptomatology or PTSD can suffer impaired functioning in home, relational, educational, and other domains.

- The risk of several other mental disorders (e.g., depression, substance abuse, conduct disorder) is substantial; suicidality is a particularly grave concern.

\section{Overview of PTSD Systematic Reviews}

The RTI-University of North Carolina Evidence-based Practice Center (EPC) conducted two protocol-based systematic reviews (with meta-analyses as appropriate) of studies involving civilian and military populations of both sexes. The prevention review dealt with adults exposed to trauma and not diagnosed with PTSD;2,4 the treatment review focused on adults with PTSD (often severe). ${ }^{3}$ We followed international procedures for conducting these two systematic reviews. ${ }^{2,3}$

We searched MEDLINE, the Cochrane Library, the Published International Literature On Traumatic Stress (PILOTS) database, EMBASE, PsycINFO, the Cumulative Index to Nursing and Allied Health Literature (CINAHL), and the Web of Science for articles published between January 1990 and July 2012 for prevention and between January 1980 and May 2012 for treatment. We searched the website of the US Food and Drug Administration and various sources for gray literature. All steps followed standard methods for selecting studies based 
on independent dual review using pre-established criteria, validating data extraction, doing two independent assessments of risk of bias of individual studies (high, medium, low), and carrying out double independent grading of strength of evidence (SOE) (high, moderate, low, insufficient).

We excluded studies assessed as high risk of bias from the main analyses. We used random-effects models to estimate pooled effects (Stata version 11.1) and did sensitivity analyses with high risk-of-bias studies. For pharmacological interventions to improve PTSD symptoms, we conducted network metaanalysis using Bayesian methods (WinBUGS Version 1.4.3, applying Markov chain Monte Carlo methods). Both reports provided extensive suggestions, based on the findings, about further research;2,3 this research brief draws from that material.

Both reviews provided extensive suggestions, based on the findings, about further research.2,3 This research brief draws from that material.

\section{Assessment of Prevention Studies}

To assess adult PTSD prevention, we included 19 trials with a range of populations who had been exposed to various psychological traumas but did not meet diagnostic criteria for PTSD. Evidence was sufficient to draw conclusions about only three interventions. These conclusions are characterized by strength of evidence (SOE) grades, which reflect how confident reviewers are about the findings reported. ${ }^{5}$

First, debriefing does not reduce either the incidence or the severity of PTSD or related psychological symptoms in civilian victims of crime, assault, or accident trauma (low SOE). The debriefing intervention in these studies was based on Mitchell's Critical Incident Stress Debriefing protocol. ${ }^{6}$

Second, in subjects with acute stress disorder (whose symptoms overlap those of PTSD and occur within the first month after trauma), brief trauma-focused cognitive behavioral therapy (CBT) was significantly more effective than supportive counseling in reducing the severity of symptoms of PTSD (moderate SOE). Pooled results did not reach statistical significance but numerically favored CBT for incidence of PTSD, depression symptom severity (both low SOE), and anxiety symptom severity (moderate SOE).

Finally, collaborative care (a complex mix of evidencebased pharmacologic and CBT components delivered by diverse types of clinicians) for a traumatic injury requiring hospitalization produced a greater decrease in PTSD symptom severity at 6,9 , and 12 months after injury than did usual care (low SOE). The efficacy of psychological interventions to prevent PTSD did not differ between men and women (low SOE).

\section{Assessment of Treatment Studies}

To assess treating adults with PTSD, we included 92 trials; patients generally had severe PTSD and mean ages in their 30 s or 40 s. Evidence supports efficacy of exposure therapy (high SOE), cognitive processing therapy, cognitive therapy, CBT-mixed therapies, eye movement desensitization and reprocessing, and narrative exposure therapy (all moderate SOE). Effect sizes for reducing PTSD symptoms were large; numbers needed to treat (NNTs) were $<4$ to achieve loss of PTSD diagnosis for all the efficacious psychological interventions except narrative exposure therapy. Fluoxetine, paroxetine, sertraline, and venlafaxine (all second-generation selective serotonin reuptake inhibitors) and topiramate (an anticonvulsant) all improved PTSD symptoms (moderate $\mathrm{SOE}$ ), and paroxetine and venlafaxine were efficacious for inducing remission (NNTs $\sim 8$; moderate SOE). Network metaanalysis of 28 trials (4,817 subjects; largely indirect evidence) found paroxetine and topiramate to be more effective than most medications for reducing PTSD symptoms (low SOE). Evidence was insufficient, however, to determine whether findings apply to all patients with PTSD or only to certain groups.

Overall, evidence about harms of interventions (for both prevention and treatment ${ }^{2,3}$ ) was insufficient to draw any conclusions.

\section{Target Areas for Research}

\section{Preventing PTSD in Adults}

Evidence supporting the effectiveness of most interventions to prevent PTSD is lacking. Because of the high number of military personnel and civilians exposed to psychological trauma and the presumed personal, societal, and financial benefits of preventing PTSD to begin with (rather than treating full cases of the disorder), more research is needed on both psychological and pharmaceutical interventions. Additional work on CBT and on complex collaborative care models is warranted, as are investigations of various pharmacologic interventions. Future research should attempt to provide more information on timing and dose of intervention-e.g., the same intervention given at different time intervals after exposure to trauma while incorporating inactive control interventions. Learning more about intervention effectiveness in various subgroups is crucial.

One key research gap is the limited ability to identify people who are at high risk of developing PTSD shortly after they have been exposed to trauma. Developing a clinical prediction rule to identify, shortly after exposure to trauma, the relatively small percentage of such individuals who will develop PTSD would be an enormous help to the field. Key variables would 
include pretrauma factors, event characteristics, and responses around the time of the traumatic event. Improving ways to identify people most at risk for developing PTSD and then evaluating the effectiveness of prevention interventions in those individuals should be the focus of future clinical and research efforts.

Although some psychological interventions produced significant decreases in traumatic stress symptoms and related psychopathology, limitations in the study methods precluded definitive guidelines for preventing PTSD in those exposed to trauma.

\section{Treating PTSD in Adults}

The evidence base was stronger for treating adults with PTSD, as several psychological and pharmacological interventions are effective. Head-to-head evidence was insufficient to determine comparative effectiveness of such therapies, so trials comparing two (or more) active interventions are still needed to fill these gaps. Key comparative trials should focus on (1) psychological treatments that have the best evidence of efficacy; (2) medications with moderate strength of evidence supporting their efficacy (fluoxetine, paroxetine, sertraline, and venlafaxine; topiramate); (3) psychological and pharmacological treatments that have the best evidence of efficacy (e.g., exposure therapy compared with paroxetine); and (4) combinations of the psychological and pharmacological treatments with the best evidence of efficacy compared with either one alone (e.g., exposure therapy plus paroxetine compared with either one alone).

\section{Assessing Harms}

Harms (adverse events and unintended negative consequences) of both psychological and pharmacological interventions are crucial future research topics. Researchers should identify potential adverse effects a priori and use or adapt validated instruments to measure them. Key outcomes to assess include mortality, suicide, suicidal ideation, self-harm behaviors, and hospitalizations.

\section{Improving Methods}

PTSD prevention or treatment studies reflect numerous deficiencies that investigators need to overcome. Being clear about the conceptual (psychological and clinical) underpinnings of tested interventions is essential. Important flaws in the literature include inadequate randomization procedures, inadequate or inappropriate statistical approaches for data analysis (e.g., lack of intention-to-treat analysis or no statistical adjustment for significant between-group differences at baseline), and lack of masking of outcome assessors.
Investigators should use better methods to minimize attrition, improve handling of missing data (e.g., multiple imputation), and obtain more complete followup data. For treatment studies, outcomes need to focus on clinically important measures-e.g., to include measures of remission or loss of a PTSD diagnosis. For prevention studies, better, clinically validated interviews and instruments are needed. Studies need to include longer followup of subjects, taking into account how long some interventions (especially medications) take before any impact might be expected.

\section{Improving Applicability of Findings}

For adult populations, virtually no information was available on differences in response to interventions for subgroups defined by sociodemographic characteristics (sex, race or ethnicity, income, or educational status), types of traumatic events and their severity, length of time since the traumatic

\section{Research Priorities for Preventing or Treating PTSD in Adults}

Prevention interventions

- Expanded studies of trauma-focused cognitive behavioral therapies and complex collaborative care models

- Trials and comparative (head-to-head) studies of both psychological and pharmaceutical interventions

Treatment comparisons

- Psychological treatments with the best evidence of efficacy

- Medications with moderate strength of evidence supporting their efficacy (fluoxetine, paroxetine, sertraline, topiramate, and venlafaxine)

- Psychological and pharmacological treatments with the best evidence of efficacy (e.g., exposure therapy compared with paroxetine)

- Combinations of the psychological and pharmacological treatments with the best evidence of efficacy compared with either one alone

\section{Crosscutting issues}

- Harms

- Subgroup analyses for patient populations defined by sociodemographic characteristics, types and severity of traumatic events, baseline levels of distress and symptoms, types of clinicians or caregivers, and settings

- Numerous methods deficiencies-e.g., specifying conceptual frameworks, improving study designs, mounting longer studies, strengthening outcome measurement, reducing attribution, handling missing data, and using appropriate statistical techniques 
event, baseline or followup severity of distress or symptoms, and coexisting conditions such as depression or anxiety. Similarly, little could be said about types of clinicians or other caregivers or settings of care.

\section{References}

1. American Psychiatric Association. Diagnostic and statistical manual of mental disorders: DSM-IV-TR. Arlington (VA): American Psychiatric Publishing; 2000.

2. Gartlehner G, Forneris CA, Brownley KA, Gaynes BN, Sonis J, Coker-Schwimmer E, Jonas DE, Greenblatt A, Wilkins TM, Woodell CL, Lohr KN. Interventions for the prevention of posttraumatic stress disorder (PTSD) in adults after exposure to psychological trauma. Rockville (MD): Agency for Healthcare Research and Quality (US); 2013 April. Comparative Effectiveness Review No. 109. Report No. 13-EHC062-EF.

3. Jonas DE, Cusack K, Forneris CA, Wilkins TM, Sonis J, Middleton JC, Feltner C, Meredith D, Cavanaugh J, Brownley KA, Olmsted KR, Greenblatt A, Weil A, Gaynes BN. Psychological and pharmacological treatments for adults with posttraumatic stress disorder (PTSD). Rockville (MD): Agency for Healthcare Research and Quality (US); 2013 April. Comparative Effectiveness Review No. 92. Report No. 13-EHC011-EF.

4. Forneris CA, Gartlehner G, Brownley KA, Gaynes BN, Sonis J, Coker-Schwimmer E, Jonas DE, Greenblatt A, Wilkins TM, Woodell C, Lohr KN. Interventions to prevent post-traumatic stress disorder: a systematic review. Am J Prev Med. 2013;44(6):635-50.
5. Owens DK, Lohr KN, Atkins D, Treadwell JR, Reston JT, Bass EB, Change S, Helfand M. AHRQ series paper 5: Grading the strength of a body of evidence when comparing medical interventionsAgency for Healthcare Research and Quality and the effective health-care program. J Clin Epidemiol. 2010 May;63(5):513-23. PMID: 19595577.

6. Mitchell JT. When disaster strikes...the critical incident stress debriefing process. JEMS. 1983;8(1):36-9.

\section{Acknowledgments}

The original systematic reviews were supported by a contract from the Agency for Healthcare Research and Quality to RTI International (the RTI-University of North Carolina Evidence-based Practice Center; contract 290-2007-10056-I). The opinions expressed in this research brief are those of the authors and do not necessarily represent the views of the Agency for Healthcare Research and Quality, the Department of Health and Human Services, or the Department of Veterans Affairs.

We thank our RTI and UNC colleagues who were coauthors on the main systematic reviews: Kim Brownley, Jamie Cavanaugh, Manny Coker-Schwimmer, Jennifer Cook-Middleton, Karen Cusack, Cynthia Feltner, Bradley Gaynes, Amy Greenblatt, Dane Meredith, Kristine Olmsted, Jeffrey Sonis, Amy Weil, and Carol Woodell. We also express appreciation to Meera Viswanathan, PhD, Director of the RTI-UNC EPC, for her steady support and leadership throughout the project, and to Loraine Monroe for close attention to document preparation efforts.

\section{About the Authors}

Catherine A. Forneris, PhD, ABPP, is a professor and licensed psychologist in the Department of Psychiatry, School of Medicine, of the University or North Carolina at Chapel Hill.

Gerald Gartlehner, MD, MPH, a senior health analyst at RTI International, serves as the associate director of the RTI-UNC Evidence-Based Practice Center (EPC) at RTI International; he is also the founding director of the Department for Evidence-Based Medicine and Clinical Epidemiology at the Danube University, Krems, Austria and directs the Austrian Cochrane Collaboration Branch there.

Daniel E. Jonas, MD, MPH, is the co-director of the RTI-UNC EPC at the Cecil G. Sheps Center for Health Services Research and assistant professor, Department of Medicine, at UNC-Chapel Hill.

Kathleen N. Lohr, PhD, MPhil, MA, is an RTI Distinguished Fellow and senior scientific advisor for the RTI-UNC EPC.
RTI Press Research Briefs and Policy Briefs are scholarly essays on policy, methods, or other topics relevant to RTI areas of research or technical focus.

RTI International, 3040 East Cornwallis Road, PO Box 12194 Research Triangle Park, NC 27709-2194 USA

$919.541 .6000 \quad$ rtipress@rti.org www.rti.org

(c)2013 Research Triangle Institute. RTI International is a trade name of Research Triangle Institute.

All rights reserved. This brief is protected by copyright. Credit must be provided to the author and source of the document when the content is quoted. Neither the document nor partial or entire reproductions may be sold without prior written permission from the publisher.

RTI Press publication RB-0005-1309

doi:10.3768/rtipress.2013.rb.0005.1309

www.rti.org/rtipress 